\section{DAP12 signaling: from immune cells to bone modeling and brain myelination}

\author{
Marco Colonna
}

Department of Pathology and Immunology, Washington University School of Medicine, St. Louis, Missouri, USA

J. Clin. Invest. 111:313-314 (2003). doi:10.1172/JCI200317745.

DAP12 (also called KARAP) is a transmembrane adapter well known for its role in transducing activation signals for an extended array of receptors in NK cells, granulocytes, monocytes/ macrophages, and DCs $(1,2)$. In this issue of the JCI, Takai and colleagues unveil that DAP12-deficient mice exhibit osteopetrosis and demyelinization of the CNS (3). Human studies have concurrently shown that genetic defects of DAP12 result in a rare syndrome characterized by bone cysts and presenile dementia $(4,5)$. Thus, DAP12 appears to be critically involved in bone modeling and brain myelination. If so, how can DAP12 regulate both immune and non-immune functions?

\section{DAP12-expressing cells in the} immune system, bones, and brain DAP12 was originally identified as a transmembrane adapter molecule that pairs with activating NK receptors, such as KIR2DS and NKG2D, and myeloid cell receptors, including SIRP$1 \beta$, MDL-1, TREM-1, -2 , and $-3(1,2)$. The cytoplasmic domain of DAP12 contains a tyrosine-based motif, which functions as a docking site for P72syk and ZAP70 tyrosine kinases. These

\footnotetext{
Address correspondence to: Department of Pathology and Immunology, Washington University School of Medicine, West Building, Room 4724, Box 8118, 660 South Euclid Avenue, St. Louis, Missouri 63110, USA. Phone: (314) 362-0367; Fax: (314) 362-4096; E-mail: mcolonna@pathology.wustl.edu. Conflict of interest: The author has declared that no conflict of interest exists. Nonstandard abbreviations used: extracellular signal-regulated kinase (ERK); Nasu-Hakola disease (NHD); polycystic lipomembranous osteodysplasia with sclerosing leukoencephalopathy (PLOSL); receptor activator of the NF-kB ligand (RANKL).
}

promote recruitment and activation of PI3K, the phospholipase $C \gamma 1$, and the $\mathrm{p} 44 / \mathrm{p} 42$ extracellular signal-regulated kinase (ERK) pathways (6). In agreement with the role of DAP12 in NK and myeloid cell activation, the initial characterization on DAP12-deficient mice revealed lack of function of activating NK cell receptors $(7,8)$, accumulation of DCs in peripheral tissues (7) and impairment of Th1 responses (8), possibly due to a reduced ability of DCs to migrate to lymph nodes and prime $\mathrm{T}$ cell responses.

This view of DAP12 as an immune signaling mediator was subsequently challenged by the observation that rare genetic mutations of human DAP12 cause no obvious immune defects but result in a syndrome characterized by bone cysts and presenile dementia called Nasu-Hakola disease (NHD), or polycystic lipomembranous osteodysplasia with sclerosing leukoencephalopathy (PLOSL) (4). Since DAP12 is expressed in cells of myeloid origin, it was suggested that DAP12 may regulate the function of osteoclasts and microglial cells, which share a myeloid origin and are critical for bone modeling and brain function, respectively. Prompted by this hypothesis, Takai and colleagues generated DAP12-deficient mice and found that they exhibit functional defects of osteoclasts and oligodendrocytes that were overlooked in previous studies (3).

\section{DAP12 as critical regulator of osteoclasts and oligodendrocytes}

The study by Takai et al shows that DAP12 protein is expressed in normal osteoclasts (3). These are polykarions of the monocyte/macrophage lineage that specialize in bone resorption (9). Normal osteoclasts derive from the fusion of mononuclear myeloid precursors in the presence of two cytokines: MCSF and a tumor necrosis factor-related protein defined as the receptor activator of the NF- $\mathrm{KB}$ ligand (RANKL) (also known as TRANCE, OPGL, and ODF) (10). However, DAP12-deficient bone marrow precursors do not differentiate in vitro into mature osteoclasts with bone resorptive function, and DAP12-deficient mice develop osteopetrosis in vivo (3). Takai and colleagues also demonstrate that DAP12 is expressed in normal oligodendrocytes and microglial cells (3). Oligodendrocytes form the myelin sheath that wraps around neural axons, enhancing nerve signal conductance in the CNS and ensuring axonal integrity. The expression of DAP12 protein in oligodendrocytes is surprising, as these cells are thought to be of neuroepithelial rather than myeloid origin (11). It is possible, however, that DAP12 is expressed only in a particular subset of oligodendrocytes, and that these might originate in the myeloid lineage. DAP12-deficiency impairs the differentiation of immature oligodendrocytes into mature myelin sheath-forming cells (3). Thus, DAP12-deficient mice show marked reduction of myelinated axons, synapse degeneration, accumulation of synaptic vesicles, especially in the medial thalamus, together with accumulation of immature oligodendrocytes in areas surrounding the thalamus. No microglial abnormalities have been detected in DAP12-deficient mice, at least at this point, despite the critical role of microglial cells in immune surveillance in the CNS (12).

Even though the clinical manifestations of DAP12-deficient mice resemble those of NHD patients, there are some significant differences. While DAP12-deficient mice exhibit osteopetrosis, NHD patients develop bone cysts filled with lipids, mainly in bones of the extremities. In DAP12-deficient mice demyelinization is concentrated in the medial thalamus. In contrast, NHD patients exhibit leukodystrophy, accumulation of lipidic material, sclerosing leukoencephalopathy, axonal loss, and massive gliosis predominant- 


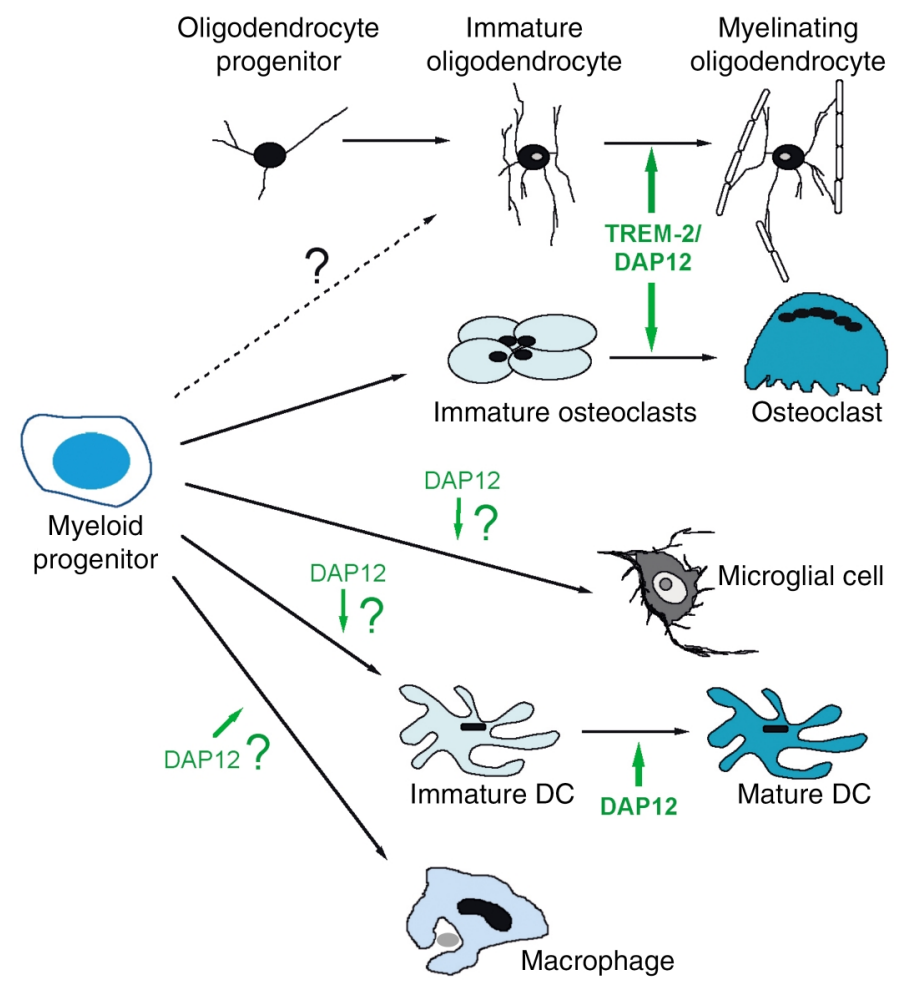

Figure 1

Hypothetical role of DAP12 in myeloid cell and oligodendrocyte development. In homeostatic conditions, the TREM-2 receptor may trigger the DAP12 signaling pathway in immature oligodendrocytes and osteoclasts, promoting their differentiation into mature cells capable of myelination and bone resorption, respectively. During inflammatory conditions, DAP12 may be solicited by different receptors, leading to differentiation and/or activation of microglial cells, macrophages, and DCs. While oligodendrocytes are thought to derive from a neuroepithelial progenitor, it is possible that DAP12-expressing oligodendrocytes may be of myeloid origin.

ly in the frontal lobes. These discrepancies may reflect the involvement of DAP12 in selected processes of bone formation and brain myelination (for example endochondral ossification or bone repair following microtraumatisms; primary myelination or remyelination during turnover or injuries), which occur at different times and places in mice and humans.

\section{The functions of DAP12-associated receptors and their ligands}

As DAP12 couples with a variety of cell surface receptors, it is important to identify the specific receptor(s) involved in the pathogenesis of the osteoclast- and oligodendrocytederived defects. Recent analysis of NHD patients with an intact DAP12 gene and normal expression levels of
DAP12 revealed loss of function mutations in TREM-2 (13). In addition, TREM-2 transcripts have been detected in human osteoclasts (13), human brain (13), and murine microglial cells (14). Thus, TREM-2 may be the DAP12-associated receptor that predominantly regulates bone modeling and CNS myelination. Future studies need to test how TREM-2/DAP12 influences osteoclast and oligodendrocyte functions. Current data suggest that TREM-2/DAP12 may guide differentiation of precursors into osteoclasts, oligodendrocytes and microglial cells, possibly by modulating responsiveness to certain cytokines or reinforcing signaling pathways triggered by cytokine receptors or integrins (Figure 1). Under homeostatic conditions, engagement of TREM-
2/DAP12 may favor osteoclast and oligodendrocyte differentiation, whereas inflammatory conditions may promote differentiation of macrophages, DCs and microglial cells through engagement of other DAP12associated receptors (Figure 1). The TREM-2/DAP12 signaling pathway may also trigger changes in actin polymerization and cytoskeleton organization, which are required for the fusion of osteoclast precursors and to ensure that the cellular processes of oligodendrocytes contact and wrap around the neuronal axons. The discovery of the ligands of TREM-2 and other DAP12associated receptors will be critical to verify these hypotheses and to precisely characterize the multiple contributions of DAP12 to immune and nonimmune functions.

1. Lanier, L.L., and Bakker, A.B. 2000. The ITAMbearing transmembrane adaptor DAP12 in lymphoid and myeloid cell function. Immunol. Today. 21:611-614.

2. Tomasello, E., et al. 1998. Gene structure, expression pattern, and biological activity of mouse killer cell activating receptor-associated protein (KARAP)/DAP-12. J. Biol. Chem. 273:34115-34119.

3. Kaifu, T., et al. 2003. Osteopetrosis and thalamic hypomyelinosis with synaptic degeneration in DAP12-deficient mice. J. Clin. Invest. 111:323-332. doi:10.1172/JCI200316923.

4. Paloneva, J., et al. 2000. Loss-of-function mutations in TYROBP (DAP12) result in a presenile dementia with bone cysts. Nat. Genet. 25:357-361.

5. Kondo, T., et al. 2002. Heterogeneity of presenile dementia with bone cysts (Nasu-Hakola disease) three genetic forms. Neurology. 59:1105-1107.

6. McVicar, D.W., et al. 1998. DAP12-mediated signal transduction in natural killer cells. A dominant role for the Syk protein-tyrosine kinase. $J$ Biol. Chem. 273:32934-32942.

7. Tomasello, E., et al. 2000. Combined natura killer cell and dendritic cell functional deficiency in KARAP/DAP12 loss-of-function mutant mice. Immunity. 13:355-364.

8. Bakker, A.B., et al. 2000. DAP12-deficient mice fail to develop autoimmunity due to impaired antigen priming. Immunity. 13:345-353.

9. Teitelbaum, S.L. 2000. Bone resorption by osteoclasts. Science. 289:1504-1508.

10. Arron, J.R., and Choi, Y. 2000. Bone versus immune system. Nature. 408:535-536.

11. Rogister, B., Ben-Hur, T., and Dubois-Dalcq, $M$ 1999. From neural stem cells to myelinating oligodendrocytes. Mol. Cell. Neurosci. 14:287-300.

12. Aloisi, F. 2001. Immune function of microglia. Glia. 36:165-179.

13. Paloneva, J., et al. 2002. Mutations in two genes encoding different subunits of a receptor signaling complex result in an identical disease phenotype. Am. J. Hum. Genet. 71:656-662.

14. Schmid, C.D., et al. 2002. Heterogeneous expres sion of the triggering receptor expressed on myeloid cells- 2 on adult murine microglia. J. Neurochem. 83:1309-1320. 